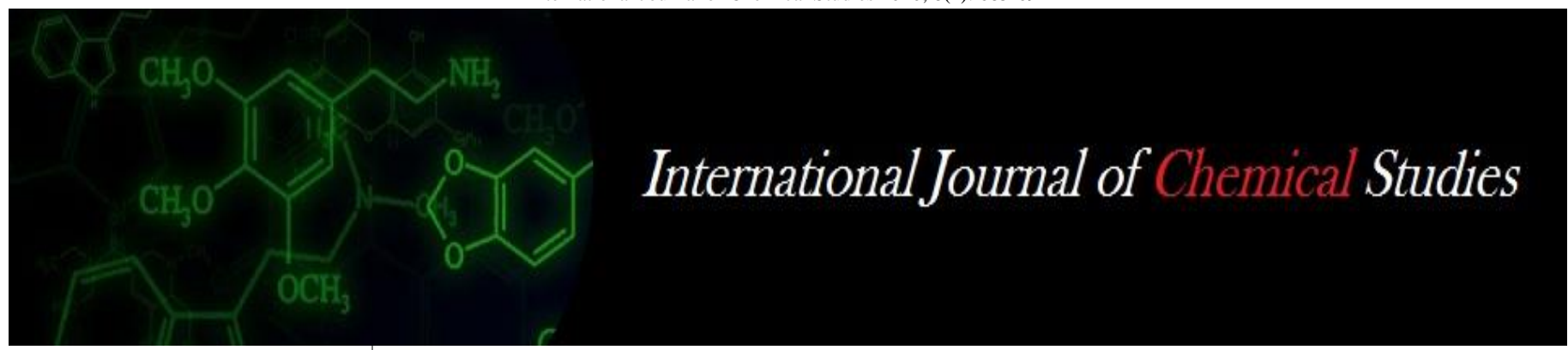

P-ISSN: 2349-8528

E-ISSN: 2321-4902

www.chemijournal.com

IJCS 2020; 8(2): 685-691

(C) 2020 IJCS

Received: 01-01-2020

Accepted: 03-02-2020

Swapan K Tripathy

Department of Agricultural

Biotechnology, College of

Agriculture, OUAT,

Bhubaneswar, Odisha, India

Suraj K Behera

Department of Agricultural

Biotechnology, College of

Agriculture, OUAT,

Bhubaneswar, Odisha, India

Dibyabharati Sahu

Department of Agricultural

Biotechnology, College of

Agriculture, OUAT,

Bhubaneswar, Odisha, India

\section{Phenotyping and association analysis of grain zinc content with agro-morphological traits in a core rice germplasm}

\author{
Swapan K Tripathy, Suraj K Behera and Dibyabharati Sahu
}

DOI: $\underline{\text { https://doi.org/10.22271/chemi.2020.v8.i2k.8845 }}$

\begin{abstract}
A set of 47 biofortified core germplasm lines of rice were estimated for grain zinc $(\mathrm{Zn})$ content to explore high $\mathrm{Zn}$ donors and to determine the degree of association of $\mathrm{Zn}$ content with agro-morphological traits including seed yield. The top $\mathrm{Zn}$ dense $(\geq 20 \mathrm{ppm})$ genotypes identified were Nagina $22(28.1 \mathrm{ppm})$, BG 102(25.8ppm), Dudh Kandar (25.6ppm), R-RHZ-7 (24.7ppm) and IR 85850-AC157-1 (24.0ppm). RRHZ-7 recorded maximum EBT $/ \mathrm{m}^{2}$ along with long panicle, while Nagina 22 maintained dwarf plant type with excellent grain fertility $(\geq 92 \%)$, thin hull and known high degree of drought tolerance. Among these, IR 85850-AC157-1(24.0ppm) and R-RHZ-7 (24.7ppm) retained high grain zinc content along with moderately high seed yield potential. Grain number/panicle $\left(\mathrm{r}=0.757^{* *}\right)$, days to flowering, days to maturity and fertility percentage revealed strong positive correlation with seed yield. Grain $\mathrm{Zn}$ content had non-significant negative association with seed yield indicating genetic enhancement for $\mathrm{Zn}$ may not have much yield penalty. Interestingly, $\mathrm{Zn}$ content revealed positive significant relationship with grain breadth, but feeble negative association with grain length and kernel length indicating that selection for bold grain types would enrich $\mathrm{Zn}$ content. The above $\mathrm{Zn}$ rich elite genotypes with desirable ancillary traits may serve as potential donors for biofortification breeding programme.
\end{abstract}

Keywords: Association analysis, grain zinc content, seed yield, zinc biofortification, rice (Oryza sativa L.)

\section{Introduction}

Malnutrition continues to be a crucial problem. Males aged between 15-74 years need $\sim 12-15$ $\mathrm{mg}$ of $\mathrm{Zn}$ daily while females aged between 12-74 years need $68 \mathrm{mg}$ of $\mathrm{Zn}$ (Sandstead, 1985) ${ }^{[27]}$. About half of world's population particularly from developing countries suffers from $\mathrm{Zn}$ deficiency and majority of them depend on rice as staple food. Rice grain is relatively low in zinc $(\mathrm{Zn})$ compared to other cereals. It is cooked and consumed as whole grain or other forms by more than half of the world population. Zn deficiency leads to loss of immunity to diseases, stunted growth, impaired learning ability, wound healing and reproduction; and increased risk of infection, DNA damage and cancer. Therefore, quality considerations are much more important than for any other food crops (Hossain et al., 2009) ${ }^{[16]}$. In India, rice is cultivated on 44.00 million hectares of area with a production of 103.00 million tons and productivity of $2.34 \mathrm{t} / \mathrm{ha}$ (Shivani et al 2019) ${ }^{[31]}$. India's current status of rice production need to be increased by $18.83 \%$ (122.4 mill tonnes) (Kumar et al. 2016) ${ }^{[20]}$ to feed estimated 1.5 billion people by 2030. With the alarming situation of malnutrition affecting human health, breeding strategies have been reoriented in last two decades to include biofortification for micronutrients, vitamins and proteins in the mainstream breeding programme. Biofortification is a sustainable genetic approach (Bouis 2002) ${ }^{[8]}$ which aims at genetic enrichment of food stuffs with vital nutrients required for human health. Grain zinc content is a complex trait accompanied by high $\mathrm{G} \times \mathrm{E}$ interaction which hinders progress in development of stable biofortified rice. There is wide variation in grain zinc concentration (14.3-31.94 ppm) (Patil et al. 2015) ${ }^{[25]}$ in brown rice suggesting tremendous scope for enrichment of this micronutrient in rice grains. Study of genetic variation to explore truly exploitable nutrient dense stable donors as parents and assessment of the relationship of iron and zinc with morpho-agronomic traits including seed yield are the initial vital steps to start any biofortification programme. Therefore, an attempt was made to identify potential $\mathrm{Zn}$-dense donors and to study the association of grain zinc content with seed yield and ancillary traits in a set of biofortified core germplasm of rice.
Corresponding Author: Swapan K Tripathy

Department of Agricultural Biotechnology, College of Agriculture, OUAT, Bhubaneswar, Odisha, India 


\section{Materials and Methods}

Plant materials and field plot technique

The experimental materials used in the present investigation comprised 47 test genotypes including 13 local land races, 23 improved biofortified breeding lines (IRRI), seven zinc rich released varieties (BRRI Dhan 62, BRRI Dhan 64, BRRI Dhan 72, DRR Dhan 45, DRR Dhan 48, DRR Dhan 49 and CGZR 1) and four high yielding ruling non-biofortified varieties (Swarna, MTU 1010, IR 64 and Sambamahsuri) of rice. These test entries were evaluated in a field trial at the Regional Research and Technology Transfer Station, Bhubaneswar following Randomized Block Design (RBD) with three replications to assess yield and ancillary traits. The crop was raised following recommended package of practices.

\section{Agro-morphological characterization}

Observations were recorded on 5 randomly selected plants from middle row of each plot for plant height $(\mathrm{cm})$, number of ear bearing tillers/hill, panicle length $(\mathrm{cm})$, grain fertility $(\%)$ and grains per panicle, while days to $50 \%$ flowering, days to maturity and seed yield (qtl/ha) were recorded on plot basis. For 100-grain weight (g), observation was taken from random sample of seeds of each plot. Dial micrometer was used to determine length and breadth of 10 grains and the respective kernels of each genotype. L/B ratios for grain and kernel were calculated taking respective mean values. Basing on grain length and L/B ratio, rice genotypes were classified into seven grain types as per Govindaswamy (1985) ${ }^{[12]}$ with minor modification as mentioned below (Table 1).

Table 1. Classification of rice genotypes based on grain type.

\begin{tabular}{|c|c|c|c|}
\hline Descriptor & $\begin{array}{c}\text { Grain } \\
\text { length(mm) }\end{array}$ & $\begin{array}{c}\text { Grain } \\
\text { Length/Breadth }\end{array}$ & Score \\
\hline Short slender(SS) & $<6.0$ & $\geq 3.0$ & 1 \\
\hline Short bold(SB) & $<6.0$ & $<2.5$ & 2 \\
\hline Medium slender(MS) & $<6.0$ & 2.5 to 3.0 & 3 \\
\hline Medium bold(MB) & $6.0-7.0$ & $2.0-2.5$ & 3.5 \\
\hline Long bold(LB) & $\geq 6.0$ & 2.5 to 3.0 & 4 \\
\hline Long slender(LS) & $\geq 6.0$ & $\geq 3.0$ & 5 \\
\hline Extra long slender(ELS) & $\geq 9.0$ & $\geq 4.0$ & 6 \\
\hline
\end{tabular}

\section{Micronutrient analysis}

Fine ground samples of each of the genotypes in three replicates were digested by di-acid mixture of nitric acid (HNO3): and perchloric acid ( $\mathrm{HClO} 4)$ in 3:2 ratio following the standard procedure of Jahan et al. (2013) ${ }^{[18]}$ with minor modification (i.e. 3:2 instead of 1:2 diacid ratio). It is based on the principle that atoms of $\mathrm{Zn}$ which normally remain in ground state, under flame condition absorb energy and subjected to radiation is proportional to the specific wavelength. The absorption of radiation is proportional to the concentration of zinc. Zinc content was estimated in the aliquot of seed extract by using Inductive Coupled PlasmaOptical Emission Spectrophotometer (ICP-OES) at 206.2nm wavelength at Central Instrumentation Facility (CIF), OUAT, Bhubaneswar. The variation in replications for each sample did not exceed $\pm 1 \mathrm{ppm}$. The mean of the three replicates were worked out to indicate $\mathrm{Zn}$-content of each genotype.

\section{Statistical analysis}

Routine statistical procedures were followed for analysis of variance and covariance as per Singh and Choudhury (1985) [32] using sample means of various traits under study. Estimates of the correlation coefficient for each pair of characters were computed following Al-Jibouri et al. (1958)
[3] to establish inter-relationship among different characters and the significance of correlation coefficients was tested by ' $\mathrm{t}$ '- test at $\mathrm{n}-2$ degrees of freedom.

\section{Results and Discussion \\ Mean performance of morpho-agronomic traits in relation to grain $\mathrm{Zn}$ content}

Rice wild relatives, upland landraces and aromatic accessions, deep water rice and coloured rice are the best sources of high grain Zn (Mallikarjuna Swamy et al. 2016) ${ }^{[21]}$. Wild species of rice e.g., O. nivara, O. rufipogon, O. latifolia, O. officinalis, and $\mathrm{O}$. granulata harbour about 2-3 fold higher grain $\mathrm{Zn}$ than cultivated rice. Besides, considerable variation for grain $\mathrm{Zn}$ content (14.5 to $35.3 \mathrm{ppm}$ ) exist in brown rice among local land races (Maganti et al 2019). In the present study, a number of IRRI breeding lines developed from different high $\mathrm{Zn}$ donors were assessed for status of grain $\mathrm{Zn}$ content and morpho-agronomic traits compared to released varieties of Bangladesh (BRRI Dhan 62, BRRI Dhan 64, BRRI Dhan 72) and India (DRR Dhan 45, DRR Dhan 48, DRR Dhan 49, CGZR 1, CGZR 3, Hurz 1 and Hurz 3) (Table $2)$. It revealed a wide array of genetic variation in maturity duration, plant types, yield potentiality and quality features including grain $\mathrm{Zn}$ content. A quest for zinc dense genotype would help breeders to combine desirable specific morphoagronomic features with high seed yield. Grain zinc content ranged from 9.2ppm in IR 97443-11-2-1-1-1-1-B to as high as 28.1ppm in Nagina 22 among 47 test genotypes including standard high yielding check varieties e.g., Swarna (17.3ppm), MTU 1010 (15.1ppm) and Sambamahsuri (12.2ppm). The top $\mathrm{Zn}$ dense( $\geq 20 \mathrm{ppm})$ genotypes identified in the present investigation were Nagina 22(28.1ppm) followed by BG 102(25.8ppm), Dudh Kandar (25.6ppm), RRHZ-7 (24.7ppm), IR 85850-AC-157-1(24.0ppm), BRRI Dhan 64(23.1ppm), IR 95133: 1-B-16-14-10-GBS-P1-23(23.1ppm), IR 95133: 1-B-16-14-10-GBS-P1-2-2(22.6ppm), DRR Dhan 45 (22.1ppm), IR 91143-AC-239-1(22.0ppm) and Sathi $(21.0 \mathrm{ppm})$. The breeding target is $28 \mathrm{ppm} \mathrm{Zn}$ in rice grain. Hence, Nagina 22 may serve as $\mathrm{Zn}$ dense donor for genetic biofortification breeding programme. Similarly, higher $\mathrm{Zn}$ content was shown to be associated with some of the aromatic rice (Gregorio 2002) ${ }^{[13]}$ and local upland rice (cv'Nam Roo': 31 ppm) (Jaksomsak et al. 2015) ${ }^{[19]}$.

Farmers are sceptic to reap more produce from each penny invested to suffice their food requirement and earning profit/unit area. Therefore, a biofortified rice variety must retain high yield potential besides being rich in micronutrient. $\mathrm{Zn}$ biofortification is targeted to breed mid-early (110125days) $\mathrm{Zn}$ rich rice genotypes. Number of effective bearing tillers (EBT), grain number/panicle, panicle length, test weight and seed fertility status determine yield potential of a test genotype. In the present investigation, R-RHZ-7 recorded maximum EBT $/ \mathrm{m}^{2}$ along with long panicle. In contrast, the top zinc rich donors "Dudh Kandar" and "BG 102" had shown tall and low tillering ability. Genotypes with good grain filling offers substantial contribution towards seed yield. BG 102 and Dudh Kandar recorded 100-grain weight even more than $3.0 \mathrm{~g}$. Fertility percentage was extremely low(54$58 \%$ ) in IR 15M 1546, Sathi and BD 102 while, few of the test genotypes maintained high fertility status $(\geq 92 \%)$ as in case of N22 and IR 95044: 8-B-5-22-19-GBS.

In the present study, IR 99642-57-1-1-1-B and BRRI Dhan 72 recorded high yield ( $\geq 46 \mathrm{q} / \mathrm{ha}$ ) followed by IR 97443-11-2-11-1-3-B, IR 97443-11-2-1-1-1-1-B, IR 91143-AC 290-1, IR 85850-AC157-1 and R-RHZ-7. The above first top four high 
yielding genotypes revealed very low to moderately low grain zinc content (14.4ppm, 17.9ppm, 12.7ppm and 9.2ppm respectively). While, the mid early maturing semi-dwarf test genotypes e.g., IR 85850-AC157-1(24.0ppm) and R-RHZ-
$7(24.7 \mathrm{ppm})$ retained high grain zinc content along with moderately high seed yield potential (around 39.0qtl/ha). Therefore, these elite genotypes have merit for commercial cultivation in irrigated and rainfed medium land ecosystem.

Table 2: Mean performance of germplasm lines for grain Zinc content and morpho-agronomic traits.

\begin{tabular}{|c|c|c|c|c|c|c|c|c|c|c|c|c|c|c|c|c|c|c|}
\hline No. & Genotype & $\begin{array}{c}\text { DF } \\
\text { (Days) }\end{array}$ & $\begin{array}{c}\text { DM } \\
\text { (days) }\end{array}$ & $\begin{array}{l}\text { PHT } \\
(\mathbf{c m})\end{array}$ & $\left|\begin{array}{c}\text { Tille } \\
\text { rs/m }\end{array}\right|$ & $\begin{array}{c}\text { PL } \\
(\mathbf{c m} .)\end{array}$ & GN/P & 100- & F\% & GL & GB & GL/ & $\begin{array}{l}\text { Grain } \\
\text { Type } \\
\text { Score } \\
\end{array}$ & KL & $\mathbf{K B}$ & $\begin{array}{c}\mathbf{K L} \\
/ \mathbf{K B}\end{array}$ & $\begin{array}{c}\mathbf{Z n} \\
(\mathbf{p p m})\end{array}$ & $\begin{array}{c}\text { Yield } \\
(\mathrm{kg} / \mathrm{ha})\end{array}$ \\
\hline 1 & BRRI Dhan 62 & 78 & 108 & 75 & 425 & 26.5 & 120 & 2.36 & 88.88 & 10.0 & 2.9 & 3.44 & 5 & 8.2 & 2.5 & 3.28 & 16.0 & 4102.6 \\
\hline 2 & BRRI Dhan 64 & 82 & 112 & 90 & 300 & 22.0 & 130 & 2.40 & 85.92 & 7.9 & 3.0 & 2.63 & 4 & 6.5 & 2.8 & 2.23 & 23.1 & 4310.2 \\
\hline 3 & BRRI Dhan 72 & 91 & 120 & 92 & 310 & 27.0 & 132 & 2.46 & 87.05 & 9.0 & 3.1 & 2.90 & 4 & 8.0 & 2.7 & 2.96 & 17.9 & 4656.5 \\
\hline 4 & BD 105 & 76 & & 90 & 290 & 23.5 & 75 & 1.88 & 58.82 & 7.0 & 2.8 & 2.50 & 4 & 6.0 & 2.8 & 2.14 & 20.0 & 3202.6 \\
\hline 5 & BG 102 & 70 & 101 & 110 & 300 & 28.8 & 78 & 3.04 & 89.28 & 9.0 & 3.0 & 3.00 & 4 & 7.5 & 2.8 & 2.67 & 25.8 & 3680.2 \\
\hline 6 & CGZR -1 & 78 & 106 & 90 & 355 & 22.5 & 90 & 2.67 & 78.26 & 9.0 & 2.8 & 3.21 & 5 & 7.5 & 2.5 & 3.00 & 21.7 & 3280.0 \\
\hline 7 & DRR Dhan 45 & 90 & 129 & 96 & 423 & 24.0 & 110 & 2.47 & 74.13 & 8.0 & 2.4 & 3.33 & 5 & 7.0 & 2.0 & 3.50 & 22.1 & 4020.4 \\
\hline 8 & DRR Dhan 48 & 97 & 127 & 92 & 420 & 26.0 & 99 & 1.75 & 81.59 & 6.0 & 2.4 & 2.50 & 3 & 5.5 & 2.0 & 2.75 & 20.0 & 4250.3 \\
\hline 9 & DRR Dhan 49 & 97 & 126 & 92 & 412 & 24.6 & 102 & 1.65 & 81.65 & 6.0 & 2.4 & 2.50 & 3 & 5.5 & 1.8 & 3.00 & 19.0 & 4280.1 \\
\hline 10 & Dudh Kandar & 74 & 123 & 130 & 294 & 29.3 & 70 & 2.97 & 72.64 & 8.9 & 3.2 & 2.78 & 4 & 7.8 & 2.2 & 3.54 & 25.6 & 2850.4 \\
\hline 11 & IR 91143-AC239-1 & 81 & 109 & 78 & 423 & 22.7 & 85 & 2.71 & 61.11 & 9.0 & 2.7 & 3.33 & 5 & 7.2 & 2.1 & 3.42 & 22.0 & 3920.0 \\
\hline 12 & IR 91143-AC290-1 & 85 & 113 & 95 & 428 & 28.2 & 90 & 2.04 & 83.29 & 9.8 & 2.8 & 3.50 & 5 & 7.5 & 2.5 & 3.00 & 20.5 & 4320.8 \\
\hline 13 & IR 91143-AC293-1 & 84 & 112 & 98 & 410 & 31.6 & 92 & 2.38 & 89.76 & 8.0 & 2.2 & 3.63 & 5 & 7.0 & 1.9 & 3.68 & 20.1 & 4260.4 \\
\hline 14 & IR 85850-AC157-1 & 98 & 127 & 93 & 412 & 25.4 & 68 & 2.18 & 74.28 & 10.0 & 3.0 & 3.33 & 5 & 8.8 & 2.8 & 3.14 & 24.0 & \\
\hline 15 & IR $82475-11$ & 94 & & 92 & 440 & 28.2 & 105 & 2.91 & 80.59 & 11.0 & 3.0 & 3.66 & 5 & 9.0 & 2.8 & 3.16 & 16.7 & \\
\hline 16 & $\begin{array}{c}\text { IR 95133: 1-B-16-14-10- } \\
\text { GBS-P1-2-2 }\end{array}$ & 94 & 123 & 100 & 342 & 28.2 & 98 & 2.49 & 63.29 & 10.0 & 2.6 & 3.84 & 5 & 9.1 & 2.1 & 4.33 & 22.6 & 3520.6 \\
\hline 17 & $\begin{array}{c}\text { IR 95133: 1-B-16-14-10- } \\
\text { GBS-P1-2-3 }\end{array}$ & 91 & 120 & 98 & 329 & 33.0 & 102 & 2.50 & 82.35 & 10.0 & 2.3 & 4.30 & 5 & 8.0 & 1.9 & 4.21 & 23.1 & 4008.4 \\
\hline 18 & $\begin{array}{l}\text { IR 95133: 1-B-16-14-10- } \\
\text { GBS-P5-1-3 }\end{array}$ & 94 & 124 & 101 & 286 & 30.6 & 105 & 2.72 & 83.56 & 10.0 & 2.4 & 4.16 & 5 & 8.0 & 2.0 & 4.00 & 17.8 & 3780.7 \\
\hline 19 & $\begin{array}{r}\text { IR 95133: } 1 \\
\text { GBS }\end{array}$ & 93 & 122 & 108 & 348 & 35.8 & 108 & 2.80 & 80.53 & 10.0 & 2.3 & 4.30 & 5 & 8.0 & 1.9 & 4.21 & 11.1 & 3820.4 \\
\hline 20 & $\begin{array}{c}\text { IR 95133: 1-B-16-14-10- } \\
\text { GBS-P6-1-5 }\end{array}$ & 86 & 114 & 102 & 362 & 29.7 & 103 & 2.56 & 81.25 & 11.0 & 2.5 & 4.40 & 5 & 9.5 & 2.0 & 4.75 & 14.8 & 4160.0 \\
\hline 21 & IR 95044: 8-B-5-22-19-GBS & 79 & 108 & 95 & 323 & 30.5 & 106 & 2.34 & 93.69 & 7.0 & 2.2 & 3.18 & 5 & 6.0 & 2.0 & 3.00 & 16.6 & 4160.6 \\
\hline 22 & IR 84847-RIL-195-1-1-1-1 & 85 & 113 & 98 & 424 & 26.6 & 100 & 2.80 & 87.35 & 10.0 & 2.7 & 3.70 & 5 & 8.2 & 2.5 & 3.28 & 15.9 & 4120.4 \\
\hline 23 & IR 99704- & 57 & 88 & 102 & 410 & 29.6 & 78 & 2.14 & 77.55 & 9.0 & 3.0 & 3.00 & 4 & 8.0 & 2.5 & 3.20 & 16.8 & 3500.2 \\
\hline 24 & IR 996 & 82 & 110 & 102 & 445 & 30.0 & 102 & 2.22 & 80.68 & 9.0 & 2.3 & 3.9 & 5 & 8.0 & 2.0 & 4.00 & 17.9 & 4208.8 \\
\hline 25 & IR 97443 & 80 & 119 & 95 & 360 & 26.4 & 118 & 1.67 & 83.91 & 8.0 & 2.2 & 3.63 & 5 & 6.5 & 1.7 & 3.82 & 9.2 & 4320.2 \\
\hline 26 & IR 97443-1 & 80 & 110 & 90 & 420 & 27.0 & 135 & 1.37 & 85.23 & 9.0 & 2.3 & 3.90 & 5 & 7.0 & 1.7 & 4.11 & 12.7 & 410.6 \\
\hline 27 & IR 962 & 85 & 114 & 90 & 430 & 24.8 & 82 & 2.10 & 65.27 & 10.0 & 2.5 & 4.00 & 5 & 8.2 & 1.8 & 4.55 & 11.9 & 3510.2 \\
\hline 28 & & 76 & 105 & 98 & 420 & 31.6 & 98 & 2.16 & 78.42 & 7.0 & 2.8 & 2.50 & 4 & 6.0 & 2.5 & 2.40 & 12.1 & 3900.0 \\
\hline 29 & & 88 & & 85 & 398 & 16.0 & 90 & 2.24 & 54.83 & 9.0 & 2.3 & 3.90 & 5 & 7.8 & 1.9 & 4.10 & 19.7 & 3460.8 \\
\hline 30 & & & & 90 & 405 & 26.4 & 80 & 2.21 & 60.00 & 9.1 & 2.4 & 3.80 & 5 & 7.8 & 2.0 & 3.90 & 13.5 & \\
\hline 31 & IR 1 & 87 & 115 & 92 & 380 & 34.0 & 86 & 2.79 & 68.42 & 10.0 & 2.5 & 4.00 & 5 & 8.2 & 2.2 & 3.72 & 12.5 & 3460.5 \\
\hline 32 & IR 9964 & 88 & 117 & 102 & 348 & 31.0 & 140 & 2.07 & 88.57 & 9.0 & 2.4 & 3.75 & 5 & 8.0 & 1.9 & 4.21 & 14.4 & 4620.4 \\
\hline 33 & & 81 & & 90 & 420 & 28.4 & 130 & 2.88 & 73.43 & 10.0 & 2.8 & 3.57 & 5 & 8.5 & 2.5 & 3.40 & 14.5 & 4190.0 \\
\hline 34 & & 82 & & 92 & 300 & 25.0 & 72 & 2.17 & 66.07 & 7.5 & 2.9 & 2.58 & 4 & 6.0 & 2.5 & 2.40 & 17.2 & 2680.4 \\
\hline 35 & U 10 & 96 & & 96 & 368 & 28.0 & 120 & 2.11 & & 8.0 & 2.3 & 3.47 & 5 & 7.2 & 2.0 & 3.60 & 15.1 & 20.6 \\
\hline 36 & M48 & 76 & 104 & 115 & 294 & 33.2 & 89 & 2.68 & 82.43 & 8.1 & 2.9 & 2.79 & 4 & 7.5 & 2.5 & 3.00 & 17.0 & 3620.0 \\
\hline 37 & 201 & 60 & 89 & 105 & 294 & 20.0 & 70 & \begin{tabular}{|l|l|}
1.87 \\
\end{tabular} & 81.96 & 7.5 & 3.0 & 2.50 & 4 & 6.0 & 2.8 & 2.14 & 17.5 & 2580.0 \\
\hline 38 & $\mathrm{~A}-22$ & 80 & 110 & 88 & 374 & 26.0 & 76 & 2.49 & 92.85 & 5.8 & 2.5 & 2.32 & 2 & 5.2 & 1.9 & 2.88 & 28.1 & 2740.2 \\
\hline 39 & & 95 & & 95 & 450 & 30.0 & 98 & 2.11 & 70.00 & 10.0 & 2.4 & 4.16 & 5 & 8.2 & 2.0 & 4.10 & 24.7 & 3850.5 \\
\hline 40 & & 73 & & 110 & 302 & \begin{tabular}{|l|}
16.0 \\
\end{tabular} & 80 & \begin{tabular}{|l|}
2.07 \\
\end{tabular} & 58.82 & 10.1 & 2.5 & 4.02 & 5 & 8.0 & 2.0 & 4.00 & 21.0 & 2460.0 \\
\hline 41 & SWARNA(Check) & 108 & 142 & 90 & 420 & 29.0 & 120 & 2.12 & 80.00 & 6.0 & 2.2 & 2.72 & 4 & 5.5 & 2.0 & 2.75 & 17.3 & 4220.9 \\
\hline 42 & SAMBAMAHSURI(Check) & 101 & 130 & 80 & 410 & 29.8 & 110 & 1.41 & 64.77 & 6.0 & 2.4 & 2.50 & 3 & 7.0 & 2.2 & 3.18 & 12.2 & 3780.0 \\
\hline 43 & $\mathrm{UI}$ & 73 & & 70 & 340 & 30.1 & 90 & 1.79 & 79.62 & 8.5 & 2.3 & 3.70 & 5 & 7.0 & 2.0 & 3.50 & 18.0 & 2830.8 \\
\hline 44 & RG-19 & 85 & 113 & 125 & 298 & 29.0 & 92 & 2.34 & 82.53 & 8.0 & 3.0 & 2.66 & 4 & 6.5 & 2.8 & 2.23 & 20.9 & 3808.2 \\
\hline 45 & & 85 & & 128 & 290 & 22.0 & 95 & 1.52 & 70.74 & 8.0 & 2.5 & 3.20 & 5 & 6.0 & 2.0 & 3.00 & 20.9 & 3610.0 \\
\hline 46 & KU- 24 & 83 & 111 & 128 & 280 & 25.0 & 90 & \begin{tabular}{|l|}
2.36 \\
\end{tabular} & \begin{tabular}{|l}
81.57 \\
\end{tabular} & 7.5 & 3.0 & \begin{tabular}{|l|}
2.50 \\
\end{tabular} & 4 & \begin{tabular}{|l|}
6.0 \\
\end{tabular} & 2.8 & \begin{tabular}{|l|}
2.14 \\
\end{tabular} & 21.9 & $\begin{array}{l}0.6 \\
3620.6\end{array}$ \\
\hline 47 & URG-30 & 74 & 104 & 102 & 275 & 27.0 & 85 & 2.21 & 79.54 & 7.0 & 2.5 & 2.80 & 4 & 5.5 & 2.2 & 2.50 & 15.0 & 2800.2 \\
\hline & Mean & 84.1 & 114 & 97.3 & 363 & 27.2 & 97.7 & 2.3 & 77.6 & 8.6 & 2.6 & 3.3 & 4.1 & 7.2 & 2.2 & 3.3 & 18.3 & 3762.2 \\
\hline \multicolumn{2}{|r|}{ Range } & $57-103$ & $88-132$ & $\begin{array}{l}70- \\
130\end{array}$ & $\begin{array}{c}275- \\
450\end{array}$ & \begin{tabular}{|c|}
$16-$ \\
35.8 \\
\end{tabular} & $\begin{array}{l}68- \\
140\end{array}$ & \begin{tabular}{|c|}
$1.4-$ \\
3.0
\end{tabular} & $\begin{array}{c}54.8- \\
93.7\end{array}$ & $\begin{array}{c}5.8- \\
11\end{array}$ & $\begin{array}{l}2.2- \\
3.2\end{array}$ & $\begin{array}{l}2.3- \\
4.4\end{array}$ & $\begin{array}{c}2- \\
5\end{array}$ & \begin{tabular}{|l|}
$5.2-$ \\
9.5
\end{tabular} & \begin{tabular}{|l|}
$1.7-$ \\
2.8
\end{tabular} & \begin{tabular}{|c|}
$2.1-$ \\
4.7
\end{tabular} & $\begin{array}{l}9.2- \\
28.1\end{array}$ & $\begin{array}{l}2460- \\
4656.5\end{array}$ \\
\hline & C.D. 0.05 & 8.6 & 7.2 & 5.8 & 36.3 & \begin{tabular}{|l|}
4.9 \\
\end{tabular} & 10.8 & \begin{tabular}{|l|}
0.4 \\
\end{tabular} & 8.2 & 2.6 & 0.16 & 0.21 & 0.56 & \multicolumn{3}{|c|}{\begin{tabular}{|l|l|l|}
0.52 & 0.09 & 0.23 \\
\end{tabular}} & 2.1 & 210.8 \\
\hline
\end{tabular}




\section{Mean performance of physical quality traits in relation to grain $\mathbf{Z n}$ content}

The top zinc dense donor Nagina 22 is a short bold type upland rice, while most of the other promising zinc dense genotypes (BG 102 :25.8ppm, Dudh Kandar :25.6ppm and IR 85850-AC157-1:24.0ppm) were characterized by long bold grains except R-RHZ-7(24.7ppm) which is a long slender type(Table 2). L/B ratio determines the grain and kernel dimension as well as grain density. Improved grain density refers to the degree of compactness of starch grains in the kernel and complete development of kernel, leaving no space between the kernel and hull. Besides, it seems to have direct bearing on improved head rice recovery (HRR). Interestingly, maximum increase in L/B ratio upon hulling was revealed in some zinc rich donors e.g., Dudh Kandar followed by Nagina 22; and it was comparable to standard quality check cv. Sambamahsuri indicating their characteristic thin and more compact hull during grain development. Besides, L/B ratio was observed to be almost equal in zinc rich rice genotypes e.g., Sathi(21.0ppm), R-RHZ-7(24.7ppm) and IR 91143AC293-1(20.1ppm) indicating proportionate minimum decrease in length and breadth after hulling leading to maintain high HRR.

\section{Association analysis \\ Inter-relationship of morpho-economic traits with seed yield}

Days to flowering, days to maturity, number of effective bearing tillers $/ \mathrm{m}^{2}$, panicle length, grain number/panicle and fertility percentage had shown positive significant correlation with seed yield. Among these, the strength of association was much higher in case of grain number/panicle( $\mathrm{r}=0.757 * *)$ followed by days to flowering, days to maturity, fertility percentage with seed yield at even $1 \%$ level of significance (Table 3). Thus, direct selection based on these component traits may offer a substantial dividend for augmentation of seed yield. Grain yield is reported to be significantly correlated with number of productive tillers/plant (Shashidhar et al., 2005, Girish et al., 2006, Monalisa et al. 2006 and Bekele et al. 2013) [29, 10], number of grains per panicle (Monalisa et al., 2006, Suman et al., 2006, Nagesh et al 2012,2013) ${ }^{[34,24]}$, panicle weight (Akinwale et al. 2011) ${ }^{[2]}$, grain fertility (Goswami et al.2000) ${ }^{[11]}$, test weight (Nagesh et al. 2012) ${ }^{[24]}$, harvest index and biological yield (Satish et al. 2003) ${ }^{[28]}$.

In the present investigation, plant height inversely correlated $(\mathrm{r}=-0.526 * *)$ with number of effective bearing tillers/ $\mathrm{m}^{2}$ at even $1 \%$ level of significance. This is due to the fact that majority of the genotypes in the present set of materials exhibited semi-dwarf plant types with high tillering ability. Besides, the number of effective bearing tillers had shown positive significant relationship with days to flowering indicating selection of semi-dwarf plant types with medium maturity duration may increase seed yield. Grain number revealed positive significant relationship with days to maturity and fertility percentage. Fertility percentage is an important component trait for realization high seed yield. It correlated positively with both panicle length and grain number as also reported by Gupta et al. (1999) ${ }^{[15]}$ and Sri Devi et al. (2019) ${ }^{[33]}$. This suggests that the genotypes with longer panicle can accommodate more number of fertile grains/panicle and thus contribute to high seed yield. This corroborates the findings of Veni et al. (2013) ${ }^{[35]}$. Besides, it is interesting to note that panicle length and fertility percentage which individually correlated positively with seed yield; had also significant inter se high positive correlation $(0.413 * *)$. Therefore, selection for any one of these characters automatically selects the other trait and thus, together could result recovery of high grain yield.

Table 3: Inter-relationship between character pairs in a set of zn rich rice genotypes.

\begin{tabular}{|c|c|c|c|c|c|c|c|c|c|c|c|c|c|c|c|c|}
\hline Character & $\begin{array}{c}\text { DF } \\
\text { (Days) }\end{array}$ & $\begin{array}{c}\text { DM } \\
\text { (days) }\end{array}$ & PHT & Tillers/m² & $\begin{array}{c}\mathbf{P L} \\
(\mathbf{c m} .)\end{array}$ & GN/P & $1000-G W$ & F\% & GL & GB & GL/GB & $\begin{array}{c}\text { Grain Type } \\
\text { Score }\end{array}$ & KL & KB & \begin{tabular}{|l|}
$\mathbf{K L}$ \\
$/ \mathbf{K B}$ \\
\end{tabular} & $\begin{array}{c}\mathbf{Z n} \\
(\mathbf{p p m})\end{array}$ \\
\hline DM & 0 & & & & & & & & & & & & & & & \\
\hline PHT & -0.19 & -0.08 & & & & & & & & & & & & & & \\
\hline Tillers/m² & $0.31 *$ & 0.26 & $-0.53 * *$ & & & & & & & & & & & & & \\
\hline \begin{tabular}{|l|} 
PL \\
\end{tabular} & 0.18 & 0.17 & 0.09 & 0.06 & & & & & & & & & & & & \\
\hline $\mathrm{G} / \mathrm{P}$ & $0.39 * *$ & $0.37 *$ & -0.22 & 0.11 & 0.22 & & & & & & & & & & & \\
\hline 100-GW & -0.06 & -0.03 & 0.18 & -0.08 & 0.27 & -0.11 & & & & & & & & & & \\
\hline $\mathrm{F} \%$ & -0.09 & -0.09 & 0.11 & -0.11 & $0.41 * *$ & $0.39 * *$ & 0.13 & & & & & & & & & \\
\hline GL & 0.01 & -0.02 & 0.03 & 0.14 & \begin{tabular}{|l|}
0.13 \\
\end{tabular} & 0.05 & $0.48 * *$ & -0.14 & & & & & & & & \\
\hline GB & $-0.39 * *$ & $-0.34 *$ & $0.29 *$ & -0.25 & -0.16 & -0.30 & $0.37 * *$ & 0.02 & 0.15 & & & & & & & \\
\hline GL/GB & \begin{tabular}{|l|}
0.21 \\
\end{tabular} & 0.16 & -0.12 & 0.23 & 0.21 & 0.21 & 0.20 & -0.14 & $0.81 * *$ & -0.46 *** & & & & & & \\
\hline Grain type- & $-0.47 * *$ & $-0.42 * *$ & $0.36^{*}$ & $-0.49 * *$ & -0.01 & -0.02 & 0.24 & -0.06 & 0.21 & $0.55 * *$ & -0.10 & & & & & \\
\hline \begin{tabular}{l|l} 
KL \\
\end{tabular} & 0.12 & 0.09 & -0.04 & 0.20 & 0.22 & 0.09 & $0.44 * *$ & -0.19 & $0.90 * *$ & \begin{tabular}{|l|}
0.10 \\
\end{tabular} & $0.75 * *$ & 0.10 & & & & \\
\hline $\mathrm{KB}$ & $-0.30 *$ & $-0.35^{*}$ & 0.18 & -0.24 & -0.11 & -0.23 & $0.31 *$ & 0.08 & \begin{tabular}{|l|}
0.04 \\
\end{tabular} & $0.88 * *$ & $-0.49 * *$ & $0.55^{* *}$ & -0.01 & & & \\
\hline \begin{tabular}{|l|} 
KL/KB \\
\end{tabular} & 0.27 & $0.29 *$ & -0.13 & 0.27 & 0.21 & 0.22 & 0.07 & -0.20 & $0.61 * *$ & $-0.55 * *$ & $0.88^{* *}$ & -0.29 & $0.69 * *$ & $-0.7 * *$ & & \\
\hline Zn content & -0.04 & 0.02 & 0.22 & -0.17 & $-0.29 *$ & $-0.38 * *$ & 0.24 & 0.01 & -0.07 & $0.32 *$ & -0.24 & -0.03 & -0.09 & 0.24 & -0.24 & \\
\hline SeedYield C & $0.50 * *$ & $0.45 * *$ & -0.15 & $0.38 * *$ & $0.33^{*}$ & $0.76^{* *}$ & -0.02 & $0.42 * *$ & 0.12 & -0.21 & 0.21 & -0.17 & 0.18 & -0.12 & 0.19 & -0.25 \\
\hline
\end{tabular}

\section{Inter se relationship with physical quality traits}

Physical quality traits determine consumers preference. Medium to long slender kernel types fit for export and most preferred by the consumers. None of the physical quality traits exhibited significant positive correlation with seed yield indicating the fact that quality traits under study had no significant contribution for realization of high seed yield. Besides, such traits revealed no significant negative correlation with seed yield, suggesting no adverse affect on seed yield while improving physical quality traits. It is expected that increase in dimension of grain (grain length and grain breadth) and kernel (kernel length and kernel breadth) would result more 100-grain weight as revealed from their inter se significant positive correlation. Grain length and kernel length had significant inter se positive correlation as also reported by Vivekanandan and Giridharan (1998) ${ }^{[36]}$ and these also maintained similar relationships with grain length/breadth and kernel length/breadth respectively. Besides, as expected grain breadth and kernel breadth had shown negative significant relationship with GL/GB and 
$\mathrm{KL} / \mathrm{KB}$ respectively indicating that bold grain and bold kernel genotypes would have less length-wise dimension. Chouhan (1996) ${ }^{[9]}$ noted similar strong association for kernel traits in some crosses of aromatic x non-aromatic varieties. Further, we noted significant positive correlation of grain length and kernel length with grain type score. This means, the long grain types inherently showed slender grain and kernel characteristics which would have relevance for export value and suitability for consumption.

\section{Correlation with grain $\mathrm{Zn}$ content}

Grain zinc content determines the success of biofortification in rice. Zinc is one of the essential micronutrients, which serves as the co-factor for more than 300 enzymes involved in the metabolism of carbohydrates, lipids, proteins, and nucleic acids; hence its importance in normal growth and development of plants and animals (Roohani et al. 2013) ${ }^{[26]}$. It negatively correlated with seed yield, though not significant at even 5\% level of significance indicating that genetic enhancement for $\mathrm{Zn}$ content would have less yield penalty. The same trend was also revealed from its regression on seed yield (fig 1). Such a feeble inverse relationship with seed yield may be ascribed to its negative relationship with panicle length and grains/panicle. Therefore, a compromise for panicle length and grain number must be taken into account while selection of plants for high grain $\mathrm{Zn}$ content. Ajmera et al (2017) [1] observed feeble non-significant positive association of grain $\mathrm{Zn}$ content with seed yield in a set of 37 rice varieties. In contrast, a number of researchers reported significant negative correlation of grain $\mathrm{Zn}$ content with seed yield in rice (Negesh et al. 2012, Naesh et al. 2013, Shivani et al. $2019^{[31]}$ and Inabangan-Asilo et al. (2019) except Sri Devi et al. (2019) ${ }^{[33]}$ who observed significant positive association between these traits in coloured rice. Genetic enhancement for grain $\mathrm{Zn}$ content was initially argued to have no/feeble yield penalty (Ashok Kumar et al. 2009) ${ }^{[6]}$. But, subsequently it was realized that over accumulation beyond $40 \mu \mathrm{g} \mathrm{Zn} / \mathrm{g}$ of rice grain (in os-NAS/IR 64 transgenic progenies) was associated with significant reductions in a number of agro-morphological traits affecting grain yield (Moreno-Moyano et al. 2016) ${ }^{[22]}$.

It was interesting to note that grain $\mathrm{Zn}$ content revealed positive significant relationship with grain breadth (0.315), but feeble negative association with grain length and kernel length indicating that bold grain types may harbour more $\mathrm{Zn}$ in the kernel. This means that the varieties which have efficient transport of zinc would have higher loading of the same to the grain resulting better grain filling and increased sink size. A set of 126 germplasm lines revealed strong negative association of $\mathrm{Zn}$ concentration with grain elongation (Anuradha et al. 2012) ${ }^{[5]}$. This was also the case in traditional short bold land races (Anandan et al, 2011) ${ }^{[4]}$ and wild rice (Gregorio et al 2000) ${ }^{[13]}$ which harbour high grain zinc content. Overall, it suggests that there is possibility of increasing $\mathrm{Zn}$ content through selection of medium length panicle containing moderate number of bold grains. A typical example is the BRRI 64 which retains similar panicle features with heavy bold grain and high grain zinc content. However, Bekele et al. (2013) ${ }^{[7]}$ showed positive relationship of grain zinc concentration with grain weight coupled with grain length.

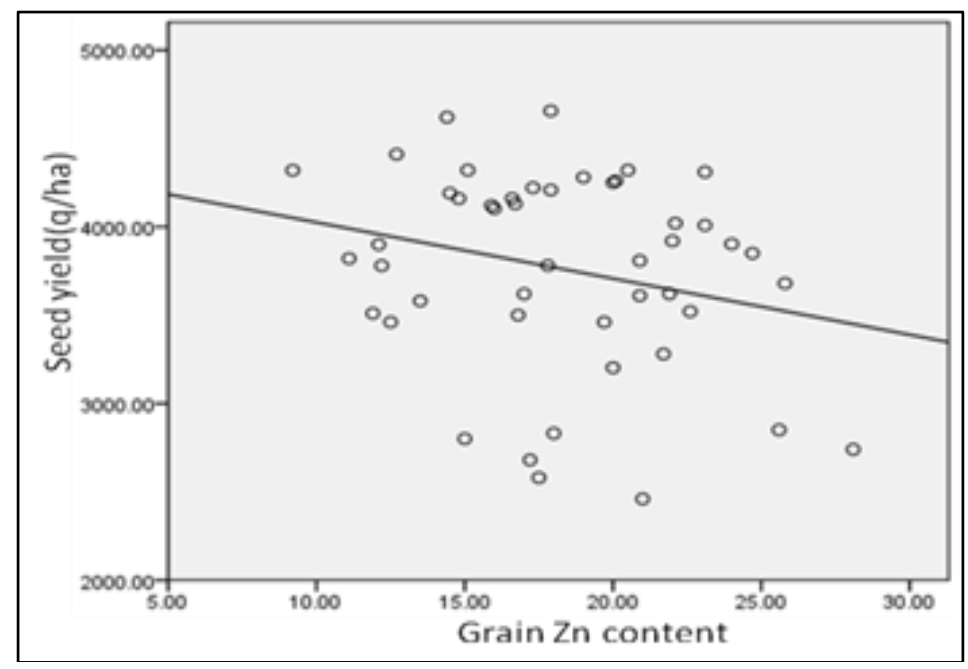

Fig 1: Relationship of grain zinc content (ppm) with seed yield (kg/ha).

\section{Conclusion}

Malnutrition continues to be a chronic health problem and even led to premature death. Rice being the major staple food, it is targeted by many researchers for $\mathrm{Zn}$ biofortification to achieve food and nutritional security. Grain $\mathrm{Zn}$ content is a highly complex trait and has high $G \times \mathrm{E}$ interaction. Exploring a stable high $\mathrm{Zn}$ donor and study of the nature of association of grain $\mathrm{Zn}$ content with seed yield and related traits are priori to biofortification programme. The high $\mathrm{Zn}$ donors with desirable morpho-agronomic traits identified in this investigation can serve as source of valuable QTLs and/or important candidate genes (Zn-transporters) for genetic enhancement of grain $\mathrm{Zn}$ content in rice. Grain number/panicle, days to flowering, days to maturity and fertility percentage had strong positive correlation with seed yield. Grain $\mathrm{Zn}$ content had significant positive association with grain breadth, but feeble negative association with grain length and kernel length. Besides, grain $\mathrm{Zn}$ content had nonsignificant negative association with seed yield indicating genetic enhancement for $\mathrm{Zn}$ may not have much yield penalty. The overall association analysis suggests that selection of semi-dwarf medium maturity plant types with more number of fertile bold grains/panicle would enrich $\mathrm{Zn}$ content in rice.

\section{Acknowledgement}

The authors highly acknowledge the Central Instrumentation Facility (CIF), OUAT for providing ICP-OES facility and IFPRI-CIAT for providing financial assistance to support the Harvest Plus Programme in collaboration with OUAT, Bhubaneswar (Odisha), India. 


\section{References}

1. Ajmera S, Kumar SS, Ravindrababu V. Character association analysis for grain iron and zinc concentrations and grain yield components in rice genotypes. Bull. Env. Pharmacol. Life Sci. 2017; 6:177-181.

2. Akinwale A, Gregorio GG, Nwilene F, Akinyele BO, Ogunbayo SA, Odiyi AC. Heritability and correlation coefficient analysis for yield and its components in rice (Oryza sativa L.). African J Plant Sci. 2011; 5:207-212.

3. Al-Jibouri HA, Mulle PA, Robinson HF. Genotypic and environmental variances and covariances in upland cotton crosses of interspecific origin. Agronomy Journal. 1958; 50:633-636.

4. Anandan A, Rajiv G, Eswaran R, Prakash M. Genetic variation and relationship between quality traits and trace elements in traditional and improved rice (Oryza sativa L.) genotypes. Journal of Food Sci. 2011; 76(4):H122H130.

5. Anuradha K, Surekha A, Rao V, Rao KV, Viraktamath BC, Sarla N. Mapping QTLs and candidate genes for iron and zinc concentrations in unpolished rice of Madhukar $\times$ Swarna RILs. Gene. 2012; 508:233-240.

6. Ashok Kumar A, Reddy BVS, Ramaiah B, Reddy PS, Sahrawat KL, Upadhyaya HD. Genetic variability and plant character association of grain $\mathrm{Fe}$ and $\mathrm{Zn}$ in selected core collection accessions of sorghum germplasm and breeding lines. SATe Journal. 2009; 7:1-4.

7. Bekele BD, Naveen GK, Rakhi S, Shashidhar HE. Genetic Evaluation of Recombinant Inbred Lines of Rice (Oryza sativa L.) for Grain Zinc Concentrations, Yield Related Traits and Identification of Associated SSR Markers. Pakistan Journal of Biological Sciences. 2013; 16:1714-1721. doi: 10.3923/pjbs.2013.1714.1721)

8. Bouis HE. Plant breeding: A new tool for fighting micronutrient malnutrition. J Nutrition. 2002; 132:491494.

9. Chouhan JS. Segregation analysis and estimation of genetic parameters for some quality traits in $F_{2}$ generation in rice. Oryza. 1996; 33:168-173.

10. Girish T, Gireesha T, Vaishali M, Hanamareddy B, Hittalmani S. Response of a new IR50/Moroberekan recombinant inbred population of rice (Oryza sativa L.) from an indica $\times$ japonica cross for growth and yield traits under aerobic conditions. Euphytica 2006; 152:149161.

11. Goswami RK, Barua KK, Pathak PK, Pathak AK. Evaluation of rice varieties for yield attributes and milling quality. JASS. 2000; 13(1):1-7.

12. Govindaswamy S. Post-harvest technology I. Quality features of rice. In: Rice Research in India. Indian Council of Agricultural Research, 1985, 627-642.

13. Gregorio GB, Senadhira D, Htut H, Graham RD. Breeding for trace mineral density in rice. Food and Nutrition Bulletin. 2000; 21(4):23-25.

14. Gregorio GB. Progress in breeding for trace minerals in staple crops. Journal of Nutrition. 2002; 132:500-502.

15. Gupta KR, Panswar DVS, Kumar R. Character association in segregating population in basmati rice. Oryza. 1999; 36(1):16-19.

16. Hossain PMD, Kawar BMD, Nahas ME. Obesity and diabetes in the developing world- a growing challenge. The New England Journal of Medicine. 2009; 356:213215.

17. Inabangan-Asilo MA, Swamy BPM, Amparado AF, Descalsota-Empleo GIL, Arocena EC, Reinke R.
Stability and G 3 E analysis of zinc-biofortified rice genotypes evaluated in diverse environments. Euphytica. 2019; 215:61.

18. Jahan GS, Hassan L, Begum SN, Islam SN. Identification of Iron rich rice genotypes in Bangladesh using chemical analysis. J. Bangladesh Agril. Univ. 2013; 11(1):73-78.

19. Jaksomsak P, Jaksomsak N, Dell B, Prom-u-thai C. Variation of seed zinc in a local upland rice germplasm from Thailand. Genetic Plant resources. 2015; 13(2):168175.

20. Kumar P, Joshi PK, Mittal S. Demand vs supply of food in India-Futuristic projection. Proceed. Of Indian Nat. Sci. Acad. 2016; 82(5):1579-1586.

21. Mallikarjuna Swamy BP, Rahman AK, Inabangan Asilo MA, Amparado A, Manito C, Mohanty $\mathrm{PC}$ et al. Advances in breeding for high grain Zinc in Rice. Rice. 2016; 9:49.

22. Moreno-Moyano LT, Bonneau JP, Sánchez-Palacios JT, Tohme J, Johnson AAT. Association of Increased Grain Iron and Zinc Concent. rations with Agro-morphological Traits of Biofortified Rice. Front. Plant Sci. 2016; 7:1463. doi: 10.3389/fpls.2016.01463

23. Nagesh Ravindra Babu V, Usha Rani G, Dayakar Reddy T, Surekha K, Vishnu Vardhan Reddy D. Association of grain iron and zinc content with yield in high yielding rice Cultivars. Oryza. 2013; 50(1):41-44.

24. Nagesh Ravindrababu V, Usharani G, Dayakar Reddy T. Grain iron and zinc association studies in rice (Oryza sativa L.) F1 progenies. Archives of Applied Science Research. 2012; 4(1):696-702.

25. Patil Ashlesha C, Shashidhar HE. Validation of Zinc transporter specific primers in rice (Oryza sativa L.) accessions grown under Aerobic condition. Research Journal of Chemistry and Environment. 2015; 20(5):3438.

26. Roohani N, Hurrell R, Kelishadi R, Schulin R. Zinc and its importance for human health: An integrative review. J Res Med Sci. 2013; 18:144-157.

27. Sandstead HH. Requirements of Zinc in human subjects. J Am Coll Nutrition. 1985; 4:73-82.

28. Satish Y, Seetha Ramaiah KV, Sree Rama Reddy N, Naidu TCM. Genetic variability, heritability and genetic advance in scented rice (Oryza sativa L.). The Andhra Agril. Journal. 2003; 50(1\&2):24-26.

29. Shashidhar HE, Pasha F, Janamatti M, Vinod MS, Kanbar A. Correlation and path co-efficient analysis in traditional cultivars and double haploid lines of rainfed lowland rice. Oryza. 2005; 42:156-159.

30. Shivani D, Cheralu C, Neeraja CN, Gouri Shankar V. Grain Zinc and Iron Association Studies in Swarna X Type 3 RIL Population of Rice. Int. J Curr. Microbiol. Appl. Sci. 2018; 7(5):708-714.

31. Shivani D, Cheralu C, Neeraja CN, Gouri Shankar V. Path coefficient analysis for grain iron and zinc concentrations and grain yield components in Swarna $x$ Type 3 RIL population of rice. International Journal of Chemical Studies. 2019; 7(3):4679-4682.

32. Singh RK, Choudhury BC. Biometrical Techniques in Genetics and Plant Breeding. International Bioscience Publisher, India, 1985, 63-68.

33. Sri Devi P, Krishna Veni B, Jyothula DPB, Sandeep Raja D. Association Studies for Yield Components, Physicochemical and Nutritional Quality Parameters in Colored Rice (Oryza sativa L.). Int. J Curr. Microbiol. Appl. Sci. 2019; 8(6):359-365. 
34. Suman A, Sreedhar N, Subba Rao LV. Correlation and path analysis of yield and its components in rice (Oryza sativa L.). Int J Trop Agric. 2006; 24:49-53.

35. Veni BK, Lakshmi BV, Ramana JV. Variability and association studies for yield components and quality parameters in rice genotypes. Journal of Rice Research. 2013; 6(2):16-23.

36. Vivekanandan P, Giridharan S. Genetic variability and character association for kernel and cooking quality in rice. Oryza. 1998; 35(3):242-245. 